\title{
Características diferenciales del bloqueo interauricular avanzado típico y atípico
}

\author{
Differential characteristics of the typical and atypical advanced interatrial block \\ Delicia Gentille-Lorente ${ }^{1 *}$ y Teresa Salvadó-Usach ${ }^{2}$ \\ ${ }^{1}$ Servicio de Cardiología; ${ }^{2}$ Servicio de Anatomía Patológica. Hospital de Tortosa Verge de la Cinta, IISPV/Tortosa, Tarragona, España
}

\section{Resumen}

Objetivo: Se denomina bloqueo interauricular avanzado $(B I A)$ a la existencia de una onda $P \geq 120$ ms y bifásica $+/-$ en las derivaciones inferiores (II-III-VF) del electrocardiograma (ECG) de superficie, el cual constituye un factor predictivo significativo del desarrollo de fibrilación auricular. En fecha reciente se han descrito cuatro patrones de BIA atípicos (BIA-At) con base en la morfología y la duración de la onda $P$, sin conocer si comparten patogenia y características clínicas similares. Método: Estudio observacional, descriptivo y retrospectivo de pacientes, visitados en el Servicio de Cardiología, en ritmo sinusal y con BIA. Las variables analizadas se obtuvieron de la historia clínica informatizada. El análisis de la onda $P$ se efectuó al aumentar el tamaño del electrocardiograma y mediante calipers electrónicos. El análisis estadístico se realizó con SPSS 19.0, con nivel de significación de $p<0.05$. Resultados: Se incluyó a 75 pacientes con media de edad de $74.4 \pm 11.7$ años, con $62.7 \%$ de varones. Se compararon los grupos de pacientes con BIA típico (BIA-T) y BIA-At. El primero se relacionó con la existencia de diabetes mellitus $(p=0.001)$, enfermedad renal crónica estadio $\geq 3(p=0.036)$, bloqueo auriculoventricular $(p=0.006)$ y una menor fracción de expulsión ventricular media $(p=0.025)$; no hubo diferencias respecto de la prevalencia de fibrilación auricular/flúter o accidente cerebrovascular. Sólo la diabetes se acompañó de riesgo de ser un BIA-T (OR: 6.4; $p=0.002$; IC 95\%: 2.0-21.1). Conclusiones: La diabetes mellitus constituye el único factor de riesgo de que un BIA sea típico. Los pacientes con BIA-T y BIA-At presentan similar prevalencia de fibrilación auricular y accidente cerebrovascular, por lo que son objeto de un mismo tratamiento clínico.

Palabras clave: Bloqueo interauricular. Bloqueo interauricular avanzado. Bloqueo interauricular de tercer grado. Bloqueo de Bachmann. Síndrome de Bayés. Fibrilación auricular.

\section{Abstract}

Objective: It is called advanced interatrial block (IAB) to the existence of a $P$ wave $\geq 120$ ms and biphasic \pm in the lower leads II-III-VF of the surface electrocardiogram (ECG), which constitutes a significant predictive factor for the development of atrial fibrillation. Recently, four patterns of atypical alAB (At-IAB) have been described based on the morphology and duration of the $P$ wave, but it's unknown if they share the same pathogenesis and clinical characteristics. Method: An observational, descriptive and retrospective study was performed with patients, visited in cardiology, who have a sinus rhythm and with alAB. The analyzed variables were obtained from the computerized clinical history. The analysis of the $P$ wave was made by

Correspondencia:

*Delicia Gentille-Lorente

E-mail: dgentille.ebre.ics@gencat.cat DOI: 10.24875/ACM.20000240
Disponible en internet: 06-02-2020 Arch Cardiol Mex. 2020;90(3):266-273 www.archivoscardiologia.com 1405-9940/@ 2020 Instituto Nacional de Cardiología Ignacio Chávez. Publicado por Permanyer. Este es un artículo open access bajo la licencia CC BY-NC-ND (http://creativecommons.org/licenses/by-nc-nd/4.0/). 
increasing the size of the ECG and by electronic calipers. Statistical analysis was performed with SPSS 19.0; level of significance: $p<0.05$. Results: $A$ total of 75 patients with an average age of $74.4 \pm 11.7$ years and with a $62.7 \%$ males, were included. It was compared the group of patients with typical alAB (T-alAB) and with At-alAB. The first one was associated with the existence of diabetes mellitus $(p=0.001)$, chronic kidney disease stage $\geq 3(p=0.036)$, atrioventricular block $(p=0.006)$ and a lower mean ventricular ejection fraction $(p=0.025)$; there were no differences regarding the prevalence of atrial fibrillation/flutter or stroke. Only diabetes was associated with the risk of T-alAB (odds ratio: $6.4 ; p=0.002 ; 95 \%$ confidence interval: 2.0-21.1). Conclusions: Diabetes mellitus is the only risk factor for an alAB to be typical. Patients with $T$-al $A B$ and At-alAB have a similar prevalence of atrial fibrillation and stroke, so they must follow the same clinical management.

Key words: Interatrial block. Advanced Interatrial Block. Bachmann's Block. Third Degree Interatrial Block. Bayés' Syndrome. Atrial Fibrillation.

\section{Introducción}

Se conoce como bloqueo interauricular a la afectación de la conducción interauricular normal a través del haz de Bachmann. Su prevalencia es alta tanto en la población general como en poblaciones específicas (pacientes hospitalizados, hipertensos o con insuficiencia cardíaca, entre otros), oscila entre el 41 y el $48.7 \%$ y es más frecuente entre los mayores de 60 a 65 años ${ }^{1}$. Cuando la conducción está interrumpida por completo, el bloqueo interauricular se considera avanzado o de tercer grado (BIA); esto supone que la activación de la aurícula izquierda (Al) ocurre en sentidos retrógrado y caudocraneal y da lugar, en el electrocardiograma (ECG) de superficie, a una onda $P \geq 120 \mathrm{~ms}$ y bifásica + - en la derivaciones inferiores II-III-VFF,3 (Fig. 1).

Aunque el BIA es una entidad independiente del crecimiento de la $\mathrm{Al}^{2}$, la mayoría de los casos se relaciona con la presencia de fibrosis y dilatación Al, de ahí que la onda $P$ sea, en principio, $\geq 120 \mathrm{~ms}$. El BIA da origen a una disfunción eléctrica y mecánica auricular que lleva al desarrollo de una "miopatía fibrótica auricular". Esta última predispone al desarrollo de arritmias supraventriculares, como el flúter $\mathrm{y}$, de modo muy especial, la fibrilación auricular (FA) ${ }^{1,3-9}$; ésto, sumado a la estasis sanguínea que ocasiona la disfunción contráctil con independencia del ritmo cardíaco existente, es lo que favorece la trombosis intraauricular ${ }^{10,11}$. Por todo ello, existe creciente evidencia de que el BIA es un factor predictivo significativo del desarrollo y recurrencia de las arritmias supraventriculares como la FA (lo que constituye el síndrome de Bayés) y, con independencia del ritmo cardíaco, también se relaciona con eventos adversos como el accidente cerebrovascular (ACV) embólico y la insuficiencia cardíaca-7,9,10,12-15.

En 2018 se describieron variantes o patrones atípicos del BIA (BIA-At) ${ }^{16}$ (Fig. 1) debidos a:

1. Variaciones en la morfología de la onda P:
- Tipo 1: P bifásica +/- en III-VF, pero en II el componente final es isodifásico $+/ 0$.

- Tipo 2: P bifásica +/- en III-VF, pero en II es trifásica +/-/+.

- Tipo 3: P bifásica +/- en II, pero en III-VF el componente inicial es isodifásico $0 /-;$ representa un diagnóstico diferencial con un ritmo de la unión pero, en el caso del BIA, la P es positiva o bifásica en las precordiales $\mathrm{V} 5-6^{17}$.

2. Variación en la duración de la onda P: si $P<120 \mathrm{~ms}$. En la actualidad se desconocen los factores que determinan el carácter típico o atípico del bloqueo y que sea atípico por las variaciones en la morfología o en la duración de la onda $\mathrm{P}$, motivo por el cual se realizó este estudio.

\section{Métodos}

Se llevó a cabo un estudio observacional, descriptivo y retrospectivo en el que se incluyó a 75 pacientes consecutivos, mayores de edad y visitados en una consulta de alta resolución de cardiología (con primera visita, ECG digitalizado, ecocardiograma y visita de resultados en un acto único), que se hallaban en ritmo sinusal y presentaban un BIA en el ECG. Las variables de estudio se obtuvieron de las historias clínicas informatizadas y fueron las que presentaban los pacientes cuando se realizó la visita; también se efectuó un "seguimiento retrospectivo" de los pacientes, es decir, la revisión de la historia clínica a fin de evaluar el desarrollo de episodios ("FA/flúter de nuevo diagnóstico" o ACV) durante el período de tiempo comprendido entre la fecha de la vista y la fecha del estudio.

Los factores de riesgo cardiovascular se definieron de acuerdo con las recomendaciones actuales, la obesidad como el índice de masa corporal $\geq 30 \mathrm{~kg} / \mathrm{m}^{2}$ y la cardiopatía isquémica como el antecedente constatado de infarto de miocardio, aterosclerosis coronaria 0 


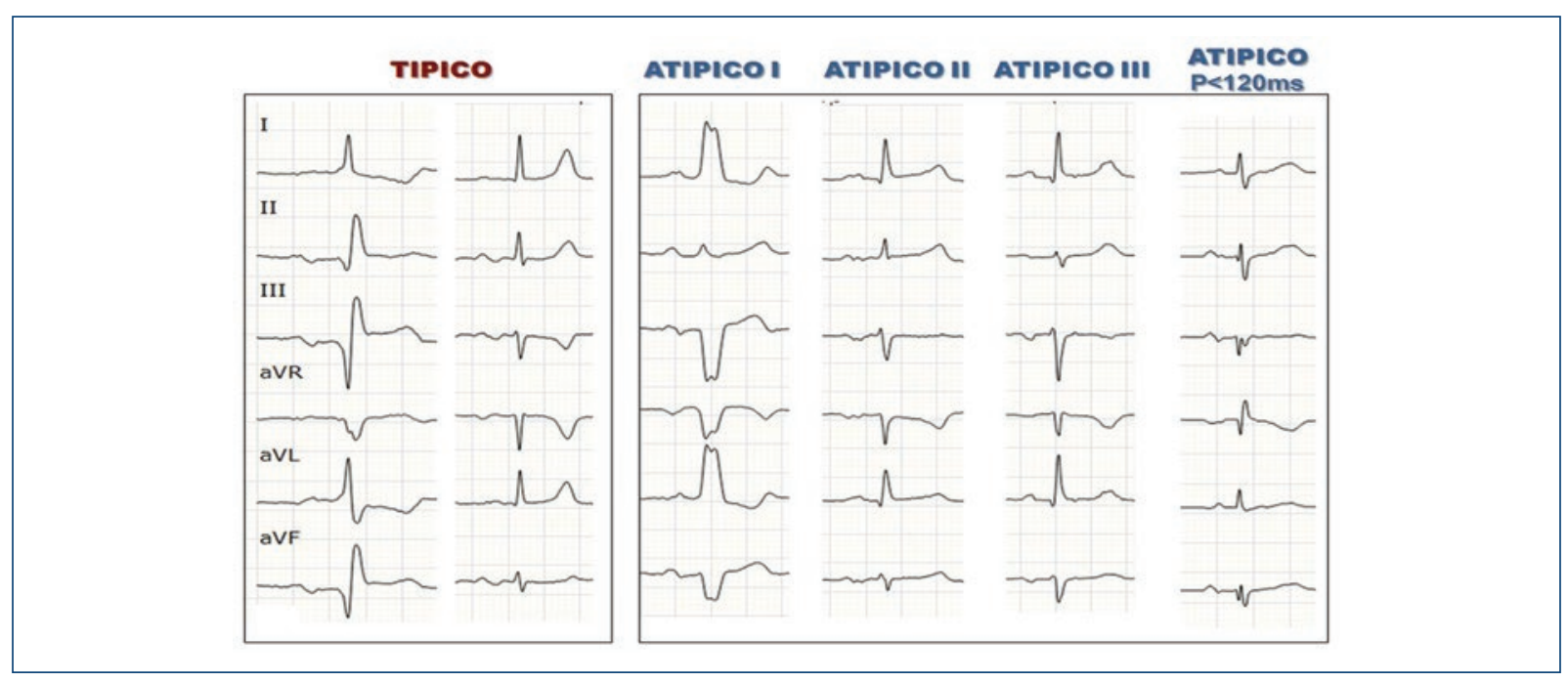

Figura 1. Presentación de los distintos tipos de BIA en las derivaciones inferiores del ECG de superficie.

revascularización miocárdica. La filtración glomerular se determinó mediante la fórmula CKD-EPI y la enfermedad renal crónica se clasificó de acuerdo con los estadios K/ DOQI.

El ECG analizado fue digital y de 12 derivaciones; los BIA típico y atípico se definieron según se estableció en los documentos de consenso correspondientes ${ }^{2,16}$; el análisis de la morfología y duración de la onda $\mathrm{P}$ se llevó a cabo de forma manual: se aumentó el tamaño del ECG y se visualizaron las seis derivaciones horizontales de manera simultánea, a fin de colocar calipers electrónicos verticales al inicio (en la primera derivación en que aparecía) y al final (en la derivación en que más tarde desaparecía) de la onda $P$ y medir el intervalo de tiempo entre ellos (en milisegundos); no se obtuvieron ni el voltaje de la $\mathrm{P}$ ni su morfología en V1.

Las ecocardiografías se realizaron con base en las recomendaciones actuales; el área $\mathrm{Al}$ considerada fue la del plano apical 4C y el volumen Al el biplano indexado por la superficie corporal. La fracción de expulsión del ventrículo izquierdo se consideró conservada si era $>51 \%$ y reducida si era $<40 \%$.

Mediante SPSS 19.0 se realizó un estudio descriptivo que expresó los resultados de las variables cuantitativas como media y desviación estándar y los de las cualitativas como porcentaje. El análisis bivariado se llevó a cabo mediante las pruebas Ji cuadrada, exacta de Fisher, t de Student y U de Mann-Whitn; se realizó regresión logística mediante análisis multivariado que incluyó a las variables con $p<0.1$. El nivel de $p$ estabecido fue $<0.05$.

\section{Resultados}

Se incluyó a un total de 75 pacientes en ritmo sinusal y con BIA, el $88 \%$ de los cuales tenía $\geq 65$ años (media de edad de $74.4 \pm 11.7$ años); los varones representaron el $62.7 \%$ de los casos; ambas características también predominaron en los distintos subgrupos de pacientes estudiados.

En una primera fase del estudio se dividió la muestra en dos grupos: pacientes con BIA típico (BIA-T) (38 casos, $50.6 \%$ ) y pacientes con BIA-At (37 casos, $49.4 \%$ ). La tabla 1 recoge las características clínicas, electrocardiográficas y ecocardiográficas de ambos grupos y el resultado del análisis bivariado; presentar un BIA-T se relacionó de modo significativo con la existencia concomitante de diabetes mellitus $(p=0.001)$, enfermedad renal crónica de estadio $\geq 3$ (filtración glomerular $<60 \mathrm{ml} / \mathrm{min})(p=0.036)$, bloqueo auriculoventricular $(p=0.006)$ y menor media de fracción de expulsión ventricular izquierda $(p=0.025)$ quedando, cerca de la significación su relación con la valvulopatía izquierda $\geq$ moderada ( $p=0.067$ ).

Sin embargo, ser un BIA-T o un BIA-At no supuso diferencias respecto de la prevalencia documentada de fibrilación auricular y flúter identificados en la fecha de la visita ni tampoco de "nuevo diagnóstico" (ocurridos desde la fecha de la visita hasta la fecha del estudio; tabla 1); el "seguimiento retrospectivo" medio fue de $18.5 \pm 14.4$ meses (mediana de 17.6 meses, moda de 17.9 meses). La prevalencia global de la FA fue similar en ambos grupos, aunque el $83.3 \%$ de los pacientes con flúter tenía un BIA-T (de los seis casos, 
Tabla 1. Principales características de los pacientes con BIA-T y BIA-At.

\begin{tabular}{|c|c|c|c|c|c|}
\hline & $\begin{array}{l}\text { BIA típico } \\
\text { (n = 38) }\end{array}$ & $\begin{array}{c}\text { BIA atípico } \\
\quad(n=37)\end{array}$ & $\begin{array}{c}\text { BIA total } \\
(n=75)\end{array}$ & $\mathbf{p}$ & 이 \\
\hline Edad (años) $\bar{X} \pm D E$ & $75.5 \pm 12.4$ & $73.2 \pm 11.0$ & $74.4 \pm 11.7$ & 0.406 & $\therefore$ \\
\hline Edad $\geq 65$ años, n (\%) & $35(92.1)$ & $31(83.8)$ & $66(88.0)$ & 0.309 & స \\
\hline Sexo: varón, n (\%) & $26(68.4)$ & $21(56.8)$ & $47(62.7)$ & 0.296 & 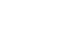 \\
\hline \multicolumn{5}{|c|}{ Antecedentes clínicos } & (2) \\
\hline HTA, n (\%) & $33(86.8)$ & $29(78.4)$ & $62(82.7)$ & 0.333 & \\
\hline Diabetes mellitus, n (\%) & $20(52.6)$ & $6(16.2)$ & $26(34.7)$ & 0.001 & to \\
\hline Dislipemia, n (\%) & $27(71.1)$ & $24(64.9)$ & $51(68.0)$ & 0.566 & 윽 \\
\hline Tabaquismo activo, n (\%) & $6(15.8)$ & $4(10.8)$ & $10(13.3)$ & 0.736 & \pm \\
\hline IMC $(\mathrm{kg} / \mathrm{m} 2) \bar{X} \pm \mathrm{DE}$ & $29.5 \pm 64$ & $29.4 \pm 4.9$ & $29.5 \pm 5.6$ & 0.939 & - \\
\hline Obesidad (IMC $\geq 30$ kg/m2), n (\%) & $18(51.4)$ & $17(47.2)$ & $35(49.3)$ & 0.723 & $\frac{4}{4}$ \\
\hline Filtración glomerular $(\mathrm{ml} / \mathrm{min}) \bar{X} \pm \mathrm{DE}$ & $66.8 \pm 25.4$ & $79.0 \pm 17.5$ & $72.9 \pm 22.5$ & 0.102 & $=1$ \\
\hline ERC estadio $\geq 2(\mathrm{FG}<90 \mathrm{ml} / \mathrm{min}), \mathrm{n}(\%)$ & $29(80.6)$ & $27(73.0)$ & $56(76.7)$ & 0.443 & 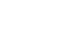 \\
\hline ERC estadio $\geq 3(\mathrm{FG}<60 \mathrm{ml} / \mathrm{min}), \mathrm{n}(\%)$ & $13(34.2)$ & $5(13.5)$ & $18(24.0)$ & 0.036 & $\stackrel{\Perp}{\varpi}$ \\
\hline EPOC, $n(\%)$ & $5(13.2)$ & $4(10.8)$ & $9(12.0)$ & 0.754 & "흥 \\
\hline SAHOS confirmado, o roncador + apneas & $4(10.5)$ & $5(13.5)$ & $9(12.0)$ & 0.736 & 인 \\
\hline Cardiopatía isquémica, n (\%) & $9(23.7)$ & $7(18.9)$ & $16(21.3)$ & 0.615 & $\bar{\Sigma}$ \\
\hline $\begin{array}{l}\text { FA/flúter conocido, n (\%) } \\
\text { FA } \\
\text { Flúter }\end{array}$ & $\begin{array}{l}9(23.7) \\
6(66.7) \\
3(33.3)\end{array}$ & $\begin{array}{l}9(24.3) \\
8(88.9) \\
1(11.1)\end{array}$ & $\begin{array}{l}18(24.0) \\
14(77.8) \\
4(22.2)\end{array}$ & $\begin{array}{l}0.948 \\
0.576\end{array}$ & $\begin{array}{l}\frac{\mathrm{J}}{2} \\
\frac{\mathrm{z}}{0} \\
\frac{0}{0}\end{array}$ \\
\hline $\begin{array}{l}\text { ACV, n (\%) } \\
\text { Ictus, n (\%) } \\
\text { AIT, n }(\%)\end{array}$ & $\begin{array}{c}5(13.2) \\
4(10.5) \\
1(2.6)\end{array}$ & $\begin{array}{l}6(16.2) \\
4(10.8) \\
2(5.4)\end{array}$ & $\begin{array}{l}11(14.7) \\
8(10.7) \\
3(4.0)\end{array}$ & $\begin{array}{l}0.708 \\
1.000 \\
0.615\end{array}$ & 흘 \\
\hline \multicolumn{5}{|c|}{ Electrocardiograma } & \\
\hline Bloqueo auriculoventricular, $\mathrm{n}(\%)$ & $16(42.1)$ & $5(13.5)$ & $21(28.0)$ & 0.006 & 8 \\
\hline Bloqueo de rama del haz de His completo, n (\%) & $16(42.1)$ & $9(24.3)$ & $25(33.3)$ & 0.102 & $\frac{1}{0}$ \\
\hline Hemibloqueo de rama, $\mathrm{n}(\%)$ & $11(28.9)$ & $6(16.2)$ & $17(22.7)$ & 0.188 & \\
\hline Índice de Cornell positivo, n (\%) & $5(13.2)$ & $5(13.5)$ & $10(13.3)$ & 1.000 & č \\
\hline Índice de Sokolo-Lyon positivo, n (\%) & $2(5.3)$ & $2(5.4)$ & $4(5.3)$ & 1.000 & 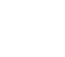 \\
\hline \multicolumn{5}{|c|}{ Ecocardiograma } & \\
\hline HVI, n (\%) & $10(26.3)$ & $6(16.2)$ & $16(21.3)$ & 0.286 & 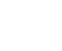 \\
\hline Tabique IV sigmoideo ( $\geq 13 \mathrm{~mm}), \mathrm{n}(\%)$ & $9(23.7)$ & $13(35.1)$ & $22(29.3)$ & 0.278 & \\
\hline Tabique IV sigmoideo $\bar{X} \pm D E$ & $16.2 \pm 2.0$ & $14.7 \pm 5.0$ & $15.4 \pm 4.0$ & 0.422 & \\
\hline Miocardiopatía (D/H/R), n (\%) & $2(5.3)$ & $2(5.4)$ & $4(5.3)$ & 1.000 & $\bar{c}$ \\
\hline FEVI $(\%) \bar{X} \pm D E$ & $58.9 \pm 8.9$ & $64.0 \pm 8.1$ & $61.4 \pm 9.4$ & 0.025 & \\
\hline $\mathrm{FEVI} \leq 51 \%, \mathrm{n}(\%)$ & $4(11.8)$ & $3(9.4)$ & $7(10.6)$ & 0.753 & \\
\hline $\mathrm{FEVI}<40 \%, \mathrm{n}(\%)$ & $3(8.8)$ & $0(0.0)$ & $3(4.5)$ & 0.239 & $\frac{5}{\hbar}$ \\
\hline Disfunción diastólica, n (\%) & $18(56.2)$ & $23(76.7)$ & & 0.090 & \\
\hline
\end{tabular}


Tabla 1. Principales características de los pacientes con BIA-T y BIA-At. (Continuación)

\begin{tabular}{|c|c|c|c|c|}
\hline & $\begin{array}{c}\text { BIA típico } \\
\text { ( } \mathrm{n}=38)\end{array}$ & $\begin{array}{c}\text { BIA atípico } \\
(\mathrm{n}=37)\end{array}$ & $\begin{array}{c}\text { BIA total } \\
(n=75)\end{array}$ & $\mathbf{p}$ \\
\hline $\begin{array}{l}\text { Grado de disfunción diastólica, } \mathrm{n}(\%) \\
<\text { moderada } \\
\geq \text { moderada }\end{array}$ & $\begin{array}{c}12(66.7) \\
6(33.3)\end{array}$ & $\begin{array}{c}17(73.9) \\
6(8.7)\end{array}$ & $\begin{array}{l}29(70.7) \\
12(29.3)\end{array}$ & 0.308 \\
\hline Al dilatada, $\mathrm{n}(\%)$ & $25(71.4)$ & $24(72.7)$ & $49(72.1)$ & 0.905 \\
\hline Área $4 \mathrm{C} \mathrm{Al}(\mathrm{cm} 2) \overline{\mathrm{X}} \pm \mathrm{DE}$ & $23.6 \pm 5.0$ & $22.8 \pm 4.6$ & $23.2 \pm 4.8$ & 0.496 \\
\hline Volumen biplano $\mathrm{Al}(\mathrm{ml} / \mathrm{cm} 2) \overline{\mathrm{X}} \pm \mathrm{DE}$ & $39.2 \pm 12.1$ & $38.8 \pm 11.7$ & $39.0 \pm 11.8$ & 0.908 \\
\hline Calcificación del anillo mitral, n (\%) & $7(20.6)$ & $6(18.2)$ & $13(19.4)$ & 0.803 \\
\hline Valvulopatía izquierda $\geq$ moderada, $\mathrm{n}(\%)$ & $4(10.5)$ & $10(27.0)$ & $14(18.7)$ & 0.067 \\
\hline Valvulopatía mitral (IM $\geq$ moderada o EM), $\mathrm{n}(\%)$ & $3(7.9)$ & $7(18.9)$ & $10(13.3)$ & 0.191 \\
\hline \multicolumn{5}{|c|}{ Seguimiento } \\
\hline $\begin{array}{l}\text { FA/flúter "de nuevo diagnóstico" desde la visita, n (\%) } \\
\text { FA } \\
\text { Flúter }\end{array}$ & $\begin{array}{l}5(13.1) \\
3(60.0) \\
2(40.0)\end{array}$ & $\begin{array}{c}2(5.4) \\
2(100.0) \\
0(0.0)\end{array}$ & $\begin{array}{l}7(9.3) \\
5(6.7) \\
2(2.7)\end{array}$ & $\begin{array}{l}0.430 \\
1.000\end{array}$ \\
\hline $\begin{array}{l}\text { FA/flúter (conocidos + "de nuevo diagnóstico"), n (\%) } \\
\text { FA } \\
\text { Flúter }\end{array}$ & $\begin{array}{l}14(36.8) \\
9(23.7) \\
5(13.2)\end{array}$ & $\begin{array}{c}11(29.7) \\
10(27.0) \\
1(2.7)\end{array}$ & $\begin{array}{c}25(33.3) \\
19(25.3) \\
6(8.0)\end{array}$ & $\begin{array}{l}0.513 \\
0.180\end{array}$ \\
\hline
\end{tabular}

Al: aurícula izquierda; AIT: accidente isquémico transitorio; BIA: bloqueo interauricular; D/H/R: dilatada/hipertrófica/restrictiva; EM: estenosis mitral; ERC: enfermedad renal crónica; FA: fibrilación auricular; FEVI: fracción de expulsión del ventrículo izquierdo biplano; FG: filtración glomerular calculada CKD-EPI; HTA: hipertensión arterial; IMC: índice de masa corporal; IM: insuficiencia mitral; IV: interventricular; VI: ventrículo izquierdo.

cinco fueron flúteres típicos levógiros). Tampoco se detectaron diferencias entre ambos grupos respecto de la prevalencia del ACV. En cuanto al bloqueo auriculoventricular, en el grupo BIA-T se identificó el $76.2 \%$ de los casos: 12 fueron BAV de primer grado, tres de segundo grado (uno tipo 2:1 y dos tipo Mobitz 1), y uno de tercer grado; en cambio, en el grupo BIA-At tres casos fueron BAV de primer grado, uno de segundo grado Mobitz 1 y uno Mobitz 2.

Tras realizar la regresión logística mediante análisis multivariado, el antecedente de diabetes mellitus fue el único acompañado de riesgo de tener un BIA-T (OR: 6.4; $p=0.002$; IC 95\%: 2.0-21.1).

En una segunda fase del estudio se analizaron las características de los 37 pacientes con BIA-At y se establecieron dos grupos: pacientes con BIA-At por variaciones en la morfología de la onda $P$ (19 casos, $51.3 \%$ ) y pacientes con BIA-At por duración de la onda P (18 casos, 48.6\%). Se analizó la relación de ambos grupos con las mismas variables consignadas en la tabla 1 de la fase 1 del estudio; la tabla 2 muestra los resultados más relevantes. En esta ocasión, sólo resultó significativa la relación entre el BIA-At por variaciones en la morfología de $\mathrm{P}$ y la existencia de una valvulopatía mitral significativa (estenosis, 0 insuficiencia $\geq$ moderada; $p=0.008$ ).

\section{Discusión}

En cuanto a la fisiopatología del BIA, la evidencia disponible señala que: a) la edad es determinante y b) el BIA constituye un factor predictivo significativo del desarrollo de arritmias supraventriculares, en particular FA, lo que constituye el síndrome de Bayés ${ }^{6,18}$.

En su génesis intervienen factores isquémicos, infiltrativos, inflamatorios y, sobre todo, degenerativos, lo que explica su distinta prevalencia según sea el grupo de edad; las comorbilidades más relacionadas con este trastorno han sido la hipertensión arterial, la diabetes mellitus y la enfermedad ateroesclerosa coronaria ${ }^{1}$, afecciones que también son muy prevalentes en los pacientes con $\mathrm{FA}^{19}$.

En este sentido, un estudio previo ${ }^{20}$ de pacientes visitados en Cardiología en que se analizaron las características distintivas de los pacientes con BIA, respecto de los que no lo tenían, ya identificó que la edad y la diabetes mellitus eran factores de riesgo del BIA, no siéndolo otros factores de riesgo más prevalentes en estos individuos (p. ej., hipertensión arterial, dislipemia y obesidad).

Con respecto a los distintos patrones de BIA-At descritos, todos tiene una condición sine qua non: el componente final de la $\mathrm{P}$ es negativo en VF, prueba de que 
Tabla 2. Principales características de los pacientes con BIA-At por morfología de la onda P y por duración de la onda P.

\begin{tabular}{|c|c|c|c|}
\hline & $\begin{array}{l}\text { BIA POR MORFOLOGÍA DE P } \\
\qquad(n=19)\end{array}$ & $\begin{array}{l}\text { BIA POR DURACIÓN DE P } \\
\qquad(n=18)\end{array}$ & $\mathbf{p}$ \\
\hline Edad (años) $\bar{X} \pm D E$ & $71.4 \pm 11.8$ & $75.1 \pm 10.2$ & 0.313 \\
\hline Sexo: varón, n (\%) & $10(52.6)$ & $11(61.1)$ & 0.603 \\
\hline HTA, n (\%) & $15(78.9)$ & $14(77.8)$ & 1.000 \\
\hline Diabetes mellitus, n (\%) & $2(10.5)$ & $4(22.2)$ & 0.405 \\
\hline Dislipemia, n (\%) & $12(63.2)$ & $12(66.7)$ & 0.823 \\
\hline Tabaquismo, n (\%) & $4(21.1)$ & $0(0.0)$ & 0.105 \\
\hline Obesidad (IMC $\left.\geq 30 \mathrm{~kg} / \mathrm{m}^{2}\right), \mathrm{n}(\%)$ & $10(52.8)$ & $7(41.2)$ & 0.491 \\
\hline ERC estadio $\geq 3(\mathrm{FG}<60 \mathrm{ml} / \mathrm{min}), \mathrm{n}(\%)$ & $2(10.5)$ & $3(16.7)$ & 0.660 \\
\hline Cardiopatía isquémica, n (\%) & $2(10.5)$ & $5(27.8)$ & 0.232 \\
\hline FA/flúter conocido, n (\%) & $7(36.8)$ & $2(11.1)$ & 0.124 \\
\hline FA/flúter de novo desde la visita, $\mathrm{n}(\%)$ & $1(5.3)$ & $1(5.5)$ & 1.000 \\
\hline ACV isquémico embólico o causa desconocida, $n(\%)$ & $3(15.8)$ & $3(16.7)$ & 1.000 \\
\hline Bloqueo auriculoventricular, n (\%) & $4(21.1)$ & $1(5.6)$ & 0.340 \\
\hline Hipertrofia VI, n (\%) & $3(15.8)$ & $3(16.7)$ & 1.000 \\
\hline FEVI VI $\bar{X} \pm D E$ & $62.6 \pm 7.3$ & $65.5 \pm 8.8$ & 0.315 \\
\hline Disfunción diastólica VI, n (\%) & $12(75.0)$ & $11(78.6)$ & 1.000 \\
\hline Al dilatada, $\mathrm{n}(\%)$ & $13(76.5)$ & $11(68.7)$ & 0.708 \\
\hline Volumen biplano indexado $\mathrm{Al}(\mathrm{ml} / \mathrm{cm} 2) \overline{\mathrm{X}} \pm \mathrm{DE}$ & $40.2 \pm 10.9$ & $37.1 \pm 13.1$ & 0.551 \\
\hline Valvulopatía cardíaca izquierda $\geq$ moderada, n (\%) & $7(36.8)$ & $0(0.0)$ & 0.008 \\
\hline
\end{tabular}

Al: aurícula izquierda; BIA: bloqueo interauricular; ERC: enfermedad renal crónica; FA: fibrilación auricular; FEVI: fracción de expulsión del ventrículo izquierdo biplano; FG: filtración glomerular calculada CKD-EPI; HTA: hipertensión arterial; IMC: índice de masa corporal; VI: ventrículo izquierdo.

la activación Al ocurre en sentido retrógrado caudocraneal; las distintas variaciones morfológicas de la onda $\mathrm{P}$ se explican por discretos cambios en la dirección de la activación Al, cuyas causas se desconocen, y la mayor brevedad de la $\mathrm{P}$ parece relacionada con un menor grado de fibrosis y dilatación Al, que se describen en pacientes jóvenes con defectos anatómicos en el tabique interauricular. Sin embargo, en conjunto y tal y como lo muestra la tabla 1, en cuanto a la edad y el sexo, los pacientes con BIA-T son sólo apenas mayores y las más de las veces corresponden a varones, mientras que aquéllos con BIA-At son algo más jóvenes, si bien la diferencia en cuanto al sexo es menor.

Al estudiar los restantes condicionantes de que un BIA sea típico $o$ atípico (fase 1 del estudio, tabla 1), no se hallaron diferencias significativas entre ambos grupos en relación con la mayor parte de las variables; sin embargo, se observa que la prevalencia de los distintos factores de riesgo cardiovascular, la de la cardiopatía isquémica y la de la enfermedad renal crónica avanzada son mayores en el grupo de BIA-T. De igual modo, en dicho grupo predominan los trastornos del sistema de conducción y alteraciones ecocardiográficas, como la hipertrofia ventricular, la disfunción sistólica, la disfunción diastólica $\geq$ moderada (es decir, con presión intrauricular elevada), y los mayores volúmenes biplanos de la Al (circunstancia que otros autores relacionan con una mayor duración de la onda P). También se detecta una relación, próxima a la significación, entre el BIA-T y la existencia de una valvulopatía izquierda relevante; dado que la valvulopatía mitral no muestra diferencias, dicha relación se basa en la mayor prevalencia de una valvulopatía aórtica en el grupo BIA-T (por lo general una estenosis aórtica pura o predominante en esta serie); dicho hallazgo, junto con el de una mayor prevalencia de bloqueo auriculoventricular en estos pacientes, 
resulta del todo lógico, dado que se trata de afecciones cuya frecuencia es directamente proporcional a la edad (que es mayor en este grupo de pacientes).

En la actualidad se considera que el BIA-At tiene las mismas implicaciones clínicas y valor predictivo de episodios adversos ${ }^{16}$ que el BIA-T, pero los resultados de la serie muestran que los pacientes con BIA-T tienen una mayor comorbilidad. No obstante, se ha detectado que sólo la diabetes posee un vínculo con riesgo de que el BIA sea típico (0R, 6.4: $p=0.002)$, lo que puede ser expresión de la existencia de una miocardiopatía diabética ${ }^{21}$ subyacente, o una relación con ella, y por tanto de un mayor grado de fibrosis auricular ${ }^{22}$. Estudios previos han demostrado que los pacientes con diabetes mellitus tipo 2 tienen en verdad un mayor volumen Al y un mayor deterioro de la distensibilidad y la contractilidad auriculares ${ }^{23}$.

También se ha demostrado con distintas técnicas de imagen (resonancia cardíaca con gadolinio, eco 3D con speckle tracking ${ }^{24,25}$ y estudios de necropsia que presentar un BIA se relaciona a menudo con la dilatación ${ }^{2}$ y la fibrosis Al; como resultado, la onda $\mathrm{P}$ es en general, $\geq$ $120 \mathrm{~ms}$. De este modo, los casos de BIA-At por duración de la $\mathrm{P}$ se consideran propios de pacientes jóvenes con determinadas alteraciones anatómicas a nivel del tabique interauricular (como una comunicación o aneurisma) ${ }^{16}$. Sin embargo, los resultados de este estudio, tal y como muestra la tabla 2, y al contrario de lo informado por otros autores, muestran que el grupo de BIA-At por la duración de la $\mathrm{P}$ no sólo presentó una media de edad considerable (75.1 \pm 10.2 años), sino que ésta fue mayor que la del grupo de atípicos por morfología de la P $(75.1 \pm 10.2$ años $)$, y además con una notoria comorbilidad. Por ejemplo, uno de los casos de BIA-AT por duración de la P fue una mujer de 73 años con un lipoma en el tabique interauricular que actuaba como "barrera" anatómica para la despolarización normal auricular.

La identificación de nuevos marcadores indicativos de la miopatía fibrótica auricular, como el $\mathrm{BIA}^{1,6,24,25}$, y por ello relacionados con riesgo de episodios cardioembólicos, puede tener importantes implicaciones clínicas: presupone que un grupo considerable de pacientes con alto riesgo de ACV podría beneficiarse de un tratamiento anticoagulante aun en ausencia de FA/flútter auriculares documentados ${ }^{22}$. En relación con esto último, se espera que el registro Bayés ${ }^{18}$, ya en marcha en la actualidad, aporte respuestas y nuevas evidencias. Futuros estudios prospectivos y con un mayor número de pacientes podrán determinar si los diferentes subtipos de BIA tienen, como se considera, un mismo significado pronóstico.

\section{Conclusiones}

El $88 \%$ de los pacientes con BIA tiene $\geq 65$ años (media de $74.4 \pm 11.7$ años) y de manera predominante corresponde a varones. La naturaleza típica del BIA se relaciona con ser diabético, tener una enfermedad renal crónica significativa, presentar un bloqueo auriculoventricular (casi siempre de primer grado) y una menor fracción de expulsión ventricular izquierda; sin embargo, sólo la diabetes mellitus constituye un factor de riesgo. Con respecto al grupo de BIA-At, se observa que las variaciones en la morfología de la onda $\mathrm{P}$ se relacionan con la existencia de una valvulopatía mitral significativa.

Pese a lo anterior, no se detectan diferencias significativas entre los grupos y subgrupos de pacientes con $\mathrm{BIA}$, respecto de la prevalencia total de FA o flútter documentados (ya sea conocidos o de nuevo diagnóstico desde la visita) o de la ACV (cardioembólico o de causa desconocida).

\section{Financiamiento}

Los autores no recibieron ningún financiamiento para la elaboración de este estudio.

\section{Conflicto de intereses}

Los autores declaran no tener ningún conflicto de intereses.

\section{Responsabilidades éticas}

Protección de personas y animales. Los autores declaran que para esta investigación no se han realizado experimentos en seres humanos ni en animales.

Confidencialidad de los datos. Los autores declaran que han seguido los protocolos de su centro de trabajo sobre la publicación de datos de pacientes.

Derecho a la privacidad y consentimiento informado. Los autores han obtenido el consentimiento informado de los pacientes o sujetos referidos en el artículo. Este documento obra en poder del autor de correspondencia.

\section{Bibliografía}

1. Baranchuk A. Interatrial block and supraventricular arrhythmias. Clinical implications of Bayés' syndrome. Cardiotext. Minneapolis, Minnesota, USA, 2017

2. Bayés de Luna A, Platonov P, Cosío FG, Cygankiewicz I, Pastore C, Baranowski R, et al. Interatrial blocks. A separate entity from left atrial enlargement: a consensus report. J Electrocardiol. 2012;45:445-51.

3. Bayés de Luna A, Cladellas M, Oter R, Torner P, Guindo J, Martí V, et al. Interatrial conduction block and retrograde activation of the left atrium and paroxysmal supraventricular tachyarrhythmia. Eur Heart J. 1988;9:1112-118. 
4. Conde D, Baranchukb A. Bloqueo interauricular como sustrato anatómico-eléctrico de arritmias supraventriculares: síndrome de Bayés. Arch Cardiol Mex. 2014; 84:32-40.

5. Conde D, Seoane L, Gysel M, Mitrione S, Bayés de Luna A, Baranchuk A. Bayés' syndrome: the association between interatrial block and supraventricular arrhythmias. Expert Rev Cardiovasc Ther. 2015;13:541-50.

6. Seoane L, Cortés M, Conde D. Update on Bayés' syndrome: the association between an interatrial block and supraventricular arrhythmias Expert Review of Cardiovascular Therapy. 2019. DOI: 10.1080/14779072. 2019.1577137.

7. Baranchuk A, Enríquez A, Antiperovitch P, Bryce Alexander, Göksel Çinier. Advanced interatrial block as a key marker for atrial fibrillation recurrence: Bayés' syndrome. J Geriatr Cardiol. 2017;14:169-73.

8. O'Neal WT, Zhang ZM, Loehr LR, Chen LY, Alonso A, Soliman EZ. Electrocardiographic advanced interatrial block and atrial fibrillation risk in the general population. Am J Cardiol. 2016;117:1755.

9. Escobar-Robledo LA, Bayés de Luna A, Lupón J, Baranchuk A, Moliner P, Martínez-Sellés $M$, et al. Advanced interatrial block predicts new-onset atrial fibrillation and ischemic stroke in patients with heart failure: the "Bayes Syndrome-HF" study. Int J Cardiol. 2018.15; 271:174-80.

10. Hirsh B, Copeland-Halperin R, HalperinJ. Fibrotic atrial cardiomyopathy, atrial fibrillation, and thromboembolism: mechanistic links and clinical inferences. J Am Coll Cadiol. 2015;65:2239-51.

11. Martínez-Selles M, Fernández-Lozano I, Baranchuck A, Bayés-Genis A, Bayés de Luna $A$. Debemos anticoagular a pacientes en alto riesgo de sufrir fibrilación auricular? Rev Esp Cardiol. 2016;69:374-6.

12. Wesley O, Kamel H, Zhu-Ming Z, Chen L, Álvaro A, Soliman E. Advanced interatrial block and ischemic Stroke. The Atherosclerosis Risk in Communities Study. Neurology. 2016;87:352-56.

13. Escobar-Robledo L, Bayés de Luna A, Lupón J, Baranchuk A, Moliner $P$, Martínez-Sellés $M$, Zamora $E$, et al. Advanced interatrial block predicts new-onset atrial fibrillation and ischemic stroke in patients with heart failure: The "Bayes' Syndrome-HF" study. Int J Cardiology. 2018; 271:174-80.
14. Arauz A, Arteaga C, Zapata-Gómez C, Ramos-Ventura C, Méndez B, Otiniano-Sifuentes $\mathrm{R}$, et al. Infarto cerebral embólico de origen no determinado: más allá de la fibrilación auricular. 2019. DOI: 10.1016/j. nrl.2019.03.008

15. Tse G, Wong CW, Gong M, Wong WT, Bazoukis G, Wong SH, et al. Predictive value of inter-atrial block for new onset or recurrent atrial fibrillation: a systematic review and meta-analysis. Int J Cardiol. 2018;250:152-6.

16. Bayés de Luna A, Escobar-Robledo LA, Aristizábal D, Weir D, Mendieta G, Massó A, et al. Atypical advanced interatrial blocks: definition and electrocardiographic recognition. J Electrocardiol. 2018;51:1091-3.

17. Bayés de Luna A, Platonov P, García-Niebla J, Baranchuk A. Atypical advanced interatrial block or junctional rhythm? J Electrocardiol. 2019;27:85-86.

18. Martínez-Sellés M, Baranchuk A, Elosua R, Bayés de Luna A. Rationale and design of the BAYES (Interatrial Block and Yearly Events) registry. Clin Cardiol. 2017; 40:196-9.

19. SunY, Hu D. The link between diabetes and atrial fibrillation: cause or correlation? J Cardiovasc Dis Res. 2010;1:10-1. 20. Gentille D, Salvadó T. Caracterización clínica, eléctrica y ecocardiográfica de los pacientes con bloqueo interauricular avanzado. Rev Esp Cardiol. 2018; 71(Supl 1):723.

21. Jia G, Hill M, Sowers J. Diabetic cardiomyopathy. An update of mechanisms contributing to this clinical entity. Circulation Research. 2018;122:624-38.

22. Calenda BW, Fuster V, Halperin JL, Granger CB. Stroke risk assessment in atrial fibrillation: risk factors and markers of atrial myopathy. Nat Rev Cardiol. 2016;13: 549-59.

23. Atas H, Kepez A, Atas DB, Kanar BG, Dervisova R, Kivrak T, et al. Effects of diabetes mellitus on left atrial volume and functions in normotensive patients without symptomatic cardiovascular disease. J Diabetes Complications. 2014;28:858-62.

24. Lacalzada-Almeida J, García-Niebla J. How to detect atrial fibrosis. J Geriatr Cardiol. 2017;14:185-94.

25. Lacalzada-Almeida J, Izquierdo-Gómez M, Belleyo-Belkasem C, Barrio-Martínez P, García-Niebla J, Elosua R, et al. Interatrial block and atrial remodeling assessed using speckle tracking echocardiography. BMC Cardiovasc Disord. 2018;18:38. 